\title{
Hilbert-Huang Transform Analysis of Storm Waves
}

\author{
J. Ortega ${ }^{1}$; George H. Smith ${ }^{2}$ \\ ${ }^{1}$ Centro de Investigación en Matemáticas, A.C., Guanajuato, Gto. México. e-mail: \\ jortega@cimat.mx \\ ${ }^{2}$ Exeter University, Cornwall Campus, U.K. e-mail: G.H.Smith@exeter.ac.uk
}

\begin{abstract}
We use the Hilbert-Huang Transform (HHT) for the spectral analysis of a North-Sea storm that took place in 1997. We look at the contribution of the different Intrinsic Mode Functions (IMF) obtained using the Empirical Mode Decomposition algorithm, and also compare the Hilbert Marginal Spectra and the classical Fourier Spectra for the data set and the corresponding IMFs. We find that the number of IMFs needed to decompose the data and the energy associated to them is different from previous studies for different sea conditions by other authors. A tentative reason for this may lie in the difference in sampling rate used.
\end{abstract}

Keywords: Hilbert-Huang Transform, spectral analysis, storm waves. 


\section{Introduction}

As is well-known, wave characteristics can show rapid changes during a storm and are also highly non-linear. Thus, to study storm waves one requires a method that takes into account these important characteristics. In 1998 Huang et al. [3] introduced a procedure for the spectral analysis of non-stationary non-linear processes which is now called the Hilbert-Huang transform (HHT). The Hilbert transform is used to define the instantaneous frequency associated to a given signal $x(t)$. If $z(t)$ is the analytic signal associated to $x(t)$, and $y(t)$ is the Hilbert transform of $x(t)$, then $z(t)=x(t)+i y(t)$. The phase function $\theta(t)=\arctan (y(t) / x(t))$ can be associated to the original signal and hence used to define the instantaneous frequency as its derivative. However, if this procedure is applied directly to a wave record, very often negative frequencies appear, which are devoid of physical meaning. This is one of the five paradoxes regarding the definition of instantaneous frequency discussed by Cohen [1].

To avoid this, the HHT algorithm decomposes the signal by a procedure called the Empirical Mode Decomposition (EMD), into the so-called Intrinsic Mode Functions (IMF). These IMFs have well-defined instantaneous frequencies, and are assumed to represent the intrinsic oscillatory modes embedded in the original signal. The Hilbert Spectrum is defined as the sum of the Hilbert spectra obtained for the individual IMFs. This method, unlike conventional Fourier decomposition, can give a very sharp time resolution for the energy-frequency content of the signal.

HHT has been used by several authors for the analysis of wave records under different situations. Veltcheva and Guedes Soares [10] analyze wave data collected at the Portuguese coast while Veltcheva [11] considered data coming form the Japanese coast. Schlurmann $[8,9]$ considered waves produced in a laboratory wave flume and sea waves also from the Sea of Japan. Dätig and Schlurmann [2] give a detailed analysis of 
the positive and negative characteristics of HHT and consider both numerically simulated nonlinear waves and waves produced in a laboratory wave flume.

In this work we consider the spectral analysis of a North-Sea storm that lasted over five days using the HHT. In the next section we give a brief description of the HilbertHuang Transform. Section 3 gives a description of the data and section 4 describes the software and parameter settings used. Section 5 analyses the results of the EMD whilst sections 6 and 7 report the results from the spectral analysis and provides comparison with the classical Fourier analysis. Finally section 8 gives some conclusion about our work.

\section{The Hilbert-Huang Transform}

We give a brief description of this algorithm. A detailed presentation can be found in the articles of Huang et al. [3, 4, 5] as well as in Huang [6,7]. The basic idea is to decompose the signal using a process known as the Empirical Mode Decomposition (EMD), which separates a time series into individual characteristic oscillatory modes known as the Intrinsic Mode Functions (IMF). It is assumed that any signal consists of different modes of oscillation based on different time scales, and each IMF represents one of these embedded oscillatory modes. IMFs must satisfy two conditions: 1) The number of local extreme points and of zero-crossings must either be equal or differ at most by one, 2) At any instant, the mean of the envelopes defined by the local maxima and minima must be zero. These two conditions are required to avoid inconsistencies in the definition of the instantaneous frequency for each IMF.

The EMD can be implemented as follows: The extreme points of the signal are identified and all maxima are joined by a cubic spline to obtain the upper envelope. In a similar way the lower envelope is obtained using the minima and all the data should fall within both envelopes. The mean $m_{1}$ is obtained as the average of both envelopes and is subtracted from the original signal to obtain a function $h_{1}=X(t)-m_{1}(t)$. If $h_{1}$ satisfies the 
criteria for an IMF, the process stops, otherwise it is repeated until one obtains a function that satisfies the criteria. This process is known as sifting and frequently has to be repeated a number of times until the first IMF is obtained. The stopping criterion for the sifting process requires that an IMF be obtained in succession a given number of times. The IMF is then subtracted from the signal and if the resulting function $r_{1}$ is not constant or a monotonic function or a function with only one extreme point, the procedure is repeated for $r_{1}$ to obtain the second IMF. This process continues until all IMFs have been extracted.

Having decomposed the signal using the EMD, the Hilbert Transform is applied to each IMF. Given a function $x(t)$ its Hilbert transform $y(t)$ is defined as

$$
y(t)=\frac{1}{\pi} \int_{-\infty}^{\infty} \frac{x(s)}{t-s} d s
$$

where the integral is taken as the Cauchy principal value. If $z(t)$ is the analytic signal associated to $x(t)$, we have for all $t, z(t)=x(t)+i y(t)=A(t) \exp \{i \theta(t)\}$ where $A(t)=\left(x^{2}(t)+y^{2}(t)\right)^{1 / 2}$ is the amplitude function and $\theta(t)=\arctan (y(t) / x(t))$ is the phase function associated to the signal. The instantaneous frequency is now defined as the derivative of the phase function $\omega(t)=\frac{d \theta(t)}{d t}$.

After decomposition into IMFs, the signal can be represented as

$$
x(t)=\operatorname{Re}\left[\sum_{j=1}^{n} A_{j}(t) \exp \left(i \int \omega_{j}(t) d t\right)\right],
$$

which can be seen as a generalized form of the Fourier decomposition for the function $x(t)$ where both amplitude and frequency are functions of time.

The time-frequency distribution of the amplitude or the amplitude squared is defined as the Hilbert amplitude spectrum or the Hilbert energy spectrum, respectively. For these spectra the time resolution can be as precise as the sampling rate of the data. The lowest 
frequency that can be obtained is $1 / \mathrm{T}$, where $\mathrm{T}$ is the duration of the record, and the highest is $1 /(5 \Delta t)$, where 5 is the minimal number of data points needed to obtain the frequency accurately and $\Delta t$ is the sampling rate. The corresponding marginal Hilbert spectrum is defined as $h(\omega)=\int_{0}^{T} H(\omega, t) d t$.

\section{Data}

Data was recorded from the North Alwyn platform situated in the northern North Sea, about 100 miles east of the Shetland Islands (608. $45^{\prime}$ North and $1^{\circ} 44.17^{\prime}$ East) in a water depth of approximately 130 metres. The data are recorded continuously, using a Thorn EMI infra-red wave height meter at $5 \mathrm{~Hz}$ and then divided into 20 minute records for which the summary statistics of $H_{s}, T_{p}$ and the spectral moments are calculated. For data with $H_{s}>3 \mathrm{~m}$ all the surface elevation records are kept. Further details are available in Wolfram et al [12]. The data set examined consists of a series of 410 records of 20 minutes, starting on November $16^{\text {th }}, 1997$. There is a 16 minutes gap of missing data

\section{Significant Wave Height}

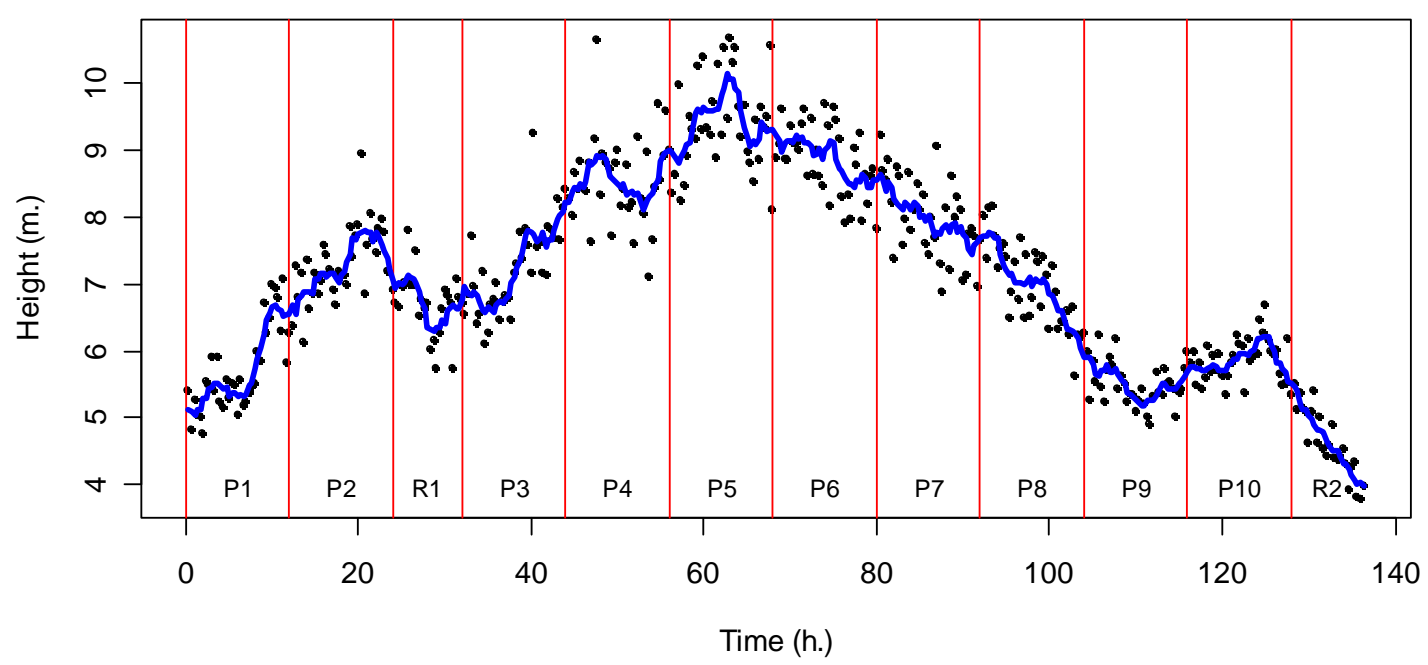

Figure 1. Significant wave height calculated every 20 minutes. The line is a 7 point moving average.

after 32 hours and 20 minutes so we have two long records, one of 97, 20-minutes intervals (32h. 20m.) and the other of 313 intervals of 20 minutes (104 h. 20m.). 
For the analysis, the data set was divided into 10 intervals of 12 hours, plus two remainders of $8 \mathrm{~h} .20 \mathrm{~min}$, denoted by $\mathrm{P}$ and $\mathrm{R}$ in figure 1 . This shows the significant wave height $\left(\mathrm{H}_{\mathrm{s}}\right)$ for the data, calculated for each $20 \mathrm{~min}$. interval, and the divisions of the data into intervals. Also shown is a 7 point moving average of the significant wave height. We concentrate on the ten 12-hour intervals. Table 1 gives a list of calculated values for the following parameters : Significant wave height $H s$, mean wave period $T_{m 01}$, spectral peak period $T_{p}$, spectral bandwidth parameter $v$ and number of IMFs that resulted after the application of the EMD.

\begin{tabular}{|c|c|c|c|c|c|c|}
\hline Period & Duration & Hs (m.) & Tm01 (s.) & Tp (s.) & v & \# IMFs \\
\hline P1 & 12 h. & 5.81 & 8.73 & 10.42 & 0.357 & 23 \\
\hline P2 & 12 h. & 7.27 & 9.67 & 11.70 & 0.370 & 24 \\
\hline R1 & 8 h. 20 m. & 6.70 & 9.52 & 12.05 & 0.336 & 18 \\
\hline P3 & 12 h. & 7.33 & 9.61 & 11.91 & 0.288 & 23 \\
\hline P4 & 12 h. & 8.58 & 10.42 & 12.57 & 0.343 & 27 \\
\hline P5 & 12 h. & 9.42 & 10.88 & 13.17 & 0.327 & 25 \\
\hline P6 & 12 h. & 8.86 & 10.57 & 13.02 & 0.244 & 23 \\
\hline P7 & 12 h. & 8.01 & 10.07 & 12.41 & 0.206 & 22 \\
\hline P8 & 12 h. & 7.00 & 9.7 & 12.10 & 0.2118 & 21 \\
\hline P9 & 12 h. & 5.54 & 8.90 & 11.31 & 0.226 & 20 \\
\hline P10 & 12 h. & 5.87 & 9.03 & 11.40 & 0.213 & 20 \\
\hline R2 & 8 h. 20 m. & 4.46 & 8.02 & 10.50 & 0.247 & 18 \\
\hline
\end{tabular}

Table 1. Some Characteristics for the Different Periods

\section{Software and Settings}

For the HHT analysis of the 12 intervals we used the Hilbert-Huang Transform Data Processing System (HHT-DPS), a software developed by NASA which has several options for the stopping criteria and the endpoint behaviour of the splines. To establish the most effective settings for our analysis we considered the 3 Endpoint Prediction choices: Pattern Prediction (PP), Copy Endpoints (CE) and Mean Prediction (MP). For each choice we used HHT-DPS on a 20-minute interval at the centre of the storm running the sifting criteria from 3 to 25. Following Veltcheva and Guedes-Soares [7], the marginal Hilbert spectra were calculated in all cases. The average of the 23 marginal 
spectra was obtained and the distance, defined as the absolute value of the difference between each marginal spectrum and the mean spectra integrated over all frequencies, was calculated for each choice of endpoint prediction setting. The best method was chosen such that this distance was minimized.

The results for the CE and MP choices were close, and are shown in figure 2, while those for PP were very irregular, showing large oscillations of the calculated distance. As can be seen, the distances for CE were almost always smaller than those of MP, showing a more consistent result. The smallest distance is obtained for the CE choice with 7 siftings, and was adopted as the standard setting for our analysis.

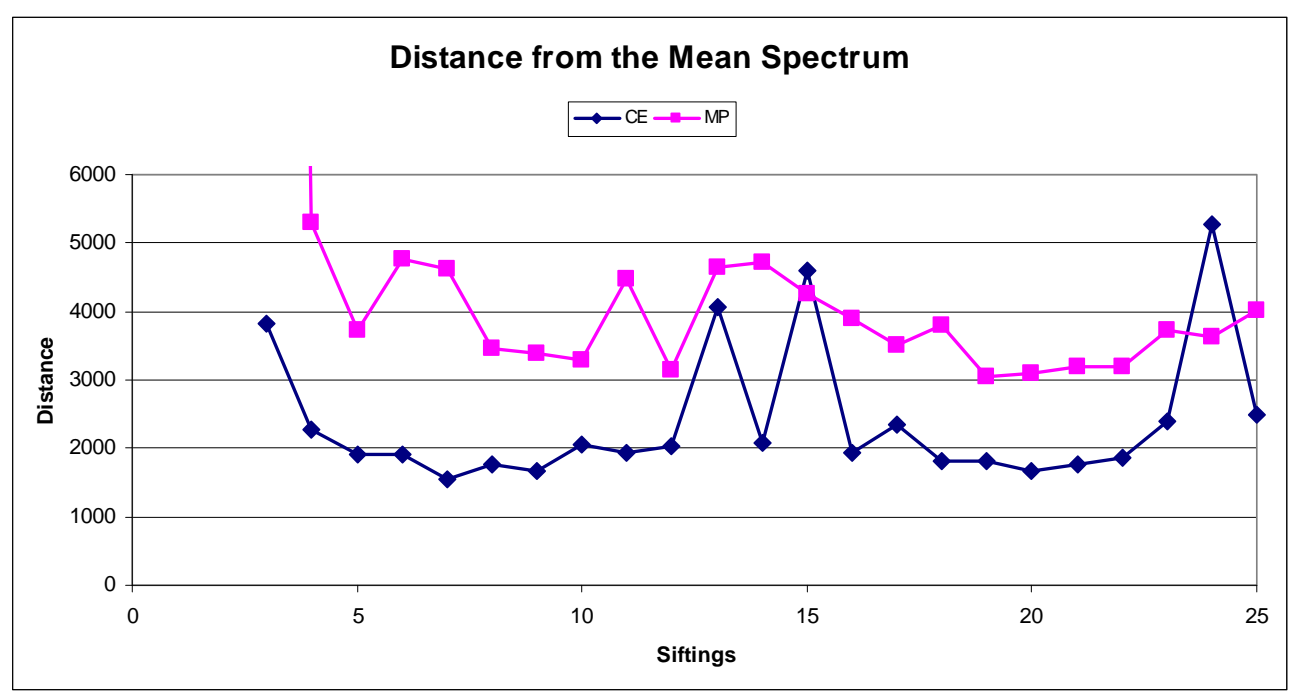

Figure 2. Distance from the mean spectrum for different siftings.

\section{Analysis of the EMD.}

The wave-elevation records for the 12-hour periods were decomposed into IMFs using HHT-DPS with the settings described above. As can be seen from Table 1, the number of IMFs needed varies from 20 to 27, depending on the specific characteristics of the interval. As an example, in Figure 3 we give the original data and the 23 IMFs obtained for a 10 -minutes interval at the beginning of the $6^{\text {th }}$ hour of period 6 (P6).

As can be seen, and as is usual in this sort of analysis, the frequency and energy content varies with each IMF. Figure 4 left shows a boxplot of the frequency content for each 

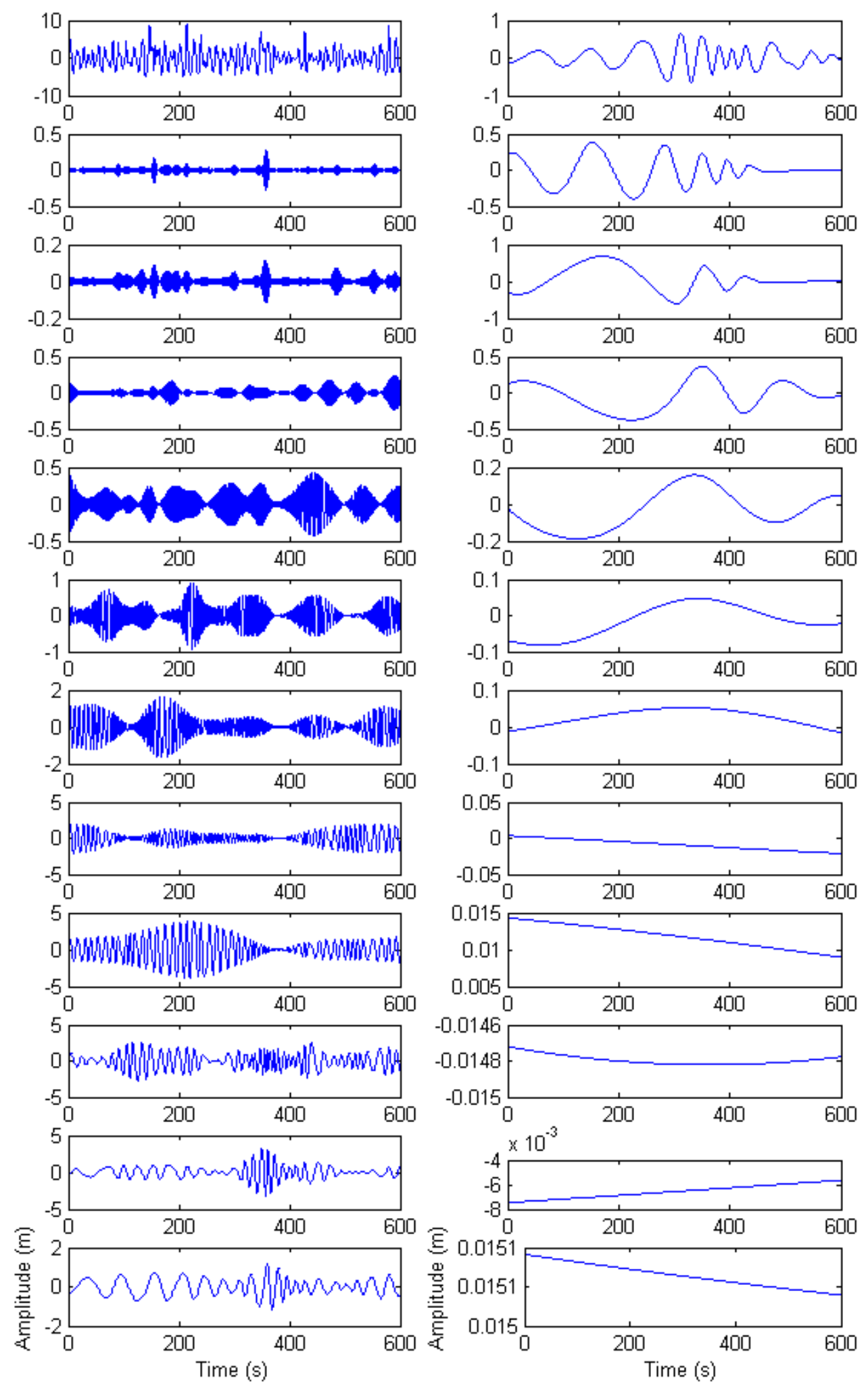

Figure 3. Original data and decomposition into IMFs for a 10-minutes interval. 
IMFs for periods P3, P6 and P9. These were chosen to represent periods when the wave height was increasing (P3), when the wave height was decreasing (P6) and finally when it decreased and then increased (P9). Higher frequencies are associated to the first IMF and decrease thereafter, although higher IMFs can still show the presence of very high frequencies. Results for the other periods were similar.

The contribution of each IMF to the total energy was determined by comparison of the variance of each IMF with the variance calculated for the original data (or equivalently, by the spectral moment of order zero $\mathrm{m}_{0}$ ) - Table 2 .

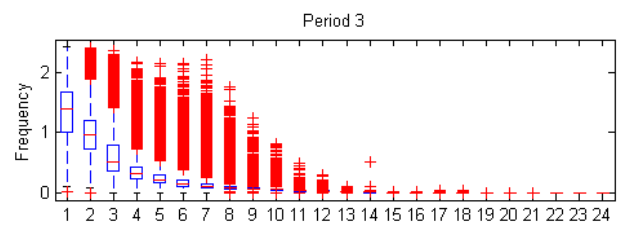

Period 6

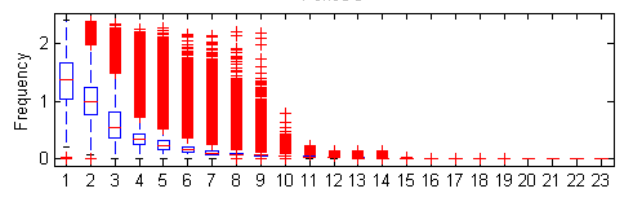

Period 9

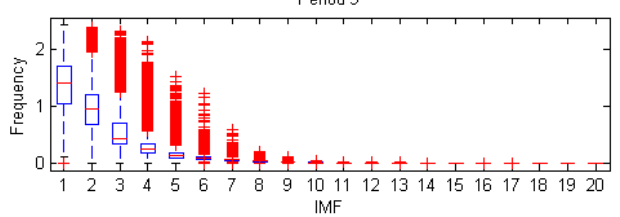

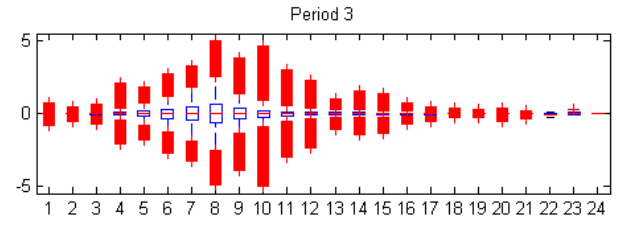

Period 6

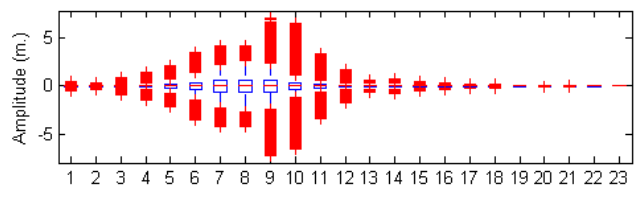

Period 9

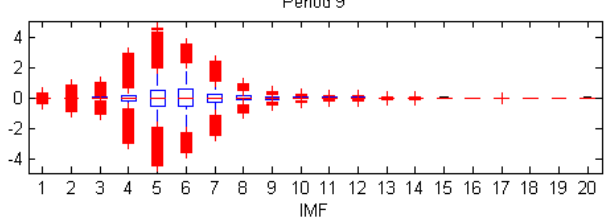

Figure 4. Boxplots for the frequency and amplitude content of each IMF, periods P3, P6 and P9.

Also, if the IMFs were orthogonal, then the sum of the variances for the IMFs would be equal to the variance of the original record, so the difference can be taken as a measure of orthogonality. Table 2 also shows the sum of the variances for each period and the difference between the two as a percentage of the true variance. The difference for all periods was always below $5 \%$ and in two cases it was below $1 \%$.

\begin{tabular}{|c|c|c|c|c|c|c|c|c|c|c|}
\hline & P1 & P2 & P3 & P4 & P5 & P6 & P7 & P8 & P9 & P10 \\
\hline Data variance $\left(\mathrm{m}^{2}\right)$ & 2.11 & 3.34 & 3.36 & 4.61 & 5.55 & 4.91 & 4.02 & 3.05 & 1.92 & 2.15 \\
\hline Sum of Variances $\left(\mathrm{m}^{2}\right)$ & 2.06 & 3.35 & 3.44 & 4.80 & 5.54 & 4.70 & 3.90 & 2.93 & 1.86 & 2.12 \\
\hline Error $(\%)$ & 2.36 & 0.28 & 2.35 & 4.32 & 0.12 & 4.26 & 2.85 & 4.16 & 3.27 & 1.47 \\
\hline
\end{tabular}

Table 2. Variance of the original data compared to the sum of variances of the IMFs 
The contribution of each IMF to the total variance also changes from period to period.

The highest variance IMF goes from IMF6 for periods P8, P9 and P10 to IMF11 for

\begin{tabular}{|r|c|r|r|r|r|r|r|r|r|r|r|}
\hline \multicolumn{3}{|c|}{ Period P3 } & \multicolumn{3}{c|}{ Period P6 } & \multicolumn{4}{c|}{ Period P9 } \\
\hline IMF & Var. & \% & Cum. \% & IMF & Var. & \multicolumn{1}{c|}{$\%$} & Cum. \% & IMF & Var. & \multicolumn{1}{c|}{$\%$} & Cum. \% \\
\hline 8 & 1.221 & 35.49 & 35.49 & 9 & 1.32 & 28.001 & 28.00 & 6 & 0.778 & 41.918 & 41.92 \\
7 & 0.592 & 17.22 & 52.71 & 7 & 1.01 & 21.383 & 49.38 & 5 & 0.687 & 37.020 & 78.94 \\
9 & 0.430 & 12.50 & 65.21 & 8 & 1.00 & 21.207 & 70.59 & 7 & 0.195 & 10.500 & 89.44 \\
\hline 6 & 0.352 & 10.22 & 75.44 & 6 & 0.50 & 10.637 & 81.23 & 4 & 0.124 & 6.656 & 96.09 \\
10 & 0.272 & 7.90 & 83.33 & 10 & 0.43 & 9.129 & 90.36 & 8 & 0.035 & 1.893 & 97.99 \\
11 & 0.145 & 4.23 & 87.56 & 5 & 0.15 & 3.132 & 93.49 & 3 & 0.014 & 0.759 & 98.75 \\
\hline 5 & 0.145 & 4.21 & 91.77 & 11 & 0.14 & 2.946 & 96.43 & 9 & 0.009 & 0.489 & 99.23 \\
4 & 0.052 & 1.52 & 93.30 & 4 & 0.05 & 0.991 & 97.43 & 10 & 0.005 & 0.273 & 99.51 \\
12 & 0.043 & 1.24 & 94.54 & 12 & 0.04 & 0.865 & 98.29 & 11 & 0.003 & 0.140 & 99.65 \\
\hline 14 & 0.032 & 0.92 & 95.45 & 14 & 0.02 & 0.449 & 98.74 & 12 & 0.002 & 0.113 & 99.76 \\
16 & 0.025 & 0.74 & 96.19 & 13 & 0.02 & 0.383 & 99.12 & 2 & 0.002 & 0.101 & 99.86 \\
15 & 0.020 & 0.57 & 96.76 & 15 & 0.01 & 0.284 & 99.41 & 13 & 0.001 & 0.062 & 99.92 \\
\hline 13 & 0.018 & 0.54 & 97.29 & 3 & 0.01 & 0.199 & 99.61 & 14 & 0.001 & 0.029 & 99.95 \\
17 & 0.017 & 0.49 & 97.79 & 16 & 0.01 & 0.173 & 99.78 & 1 & 0.000 & 0.026 & 99.98 \\
23 & 0.012 & 0.34 & 98.13 & 17 & 0.00 & 0.099 & 99.88 & 15 & 0.000 & 0.008 & 99.99 \\
\hline 18 & 0.012 & 0.34 & 98.48 & 18 & 0.00 & 0.047 & 99.92 & 16 & 0.000 & 0.005 & 99.99 \\
3 & 0.012 & 0.34 & 98.82 & 1 & 0.00 & 0.020 & 99.94 & 17 & 0.000 & 0.003 & 99.995 \\
20 & 0.011 & 0.33 & 99.15 & 2 & 0.00 & 0.014 & 99.96 & 20 & 0.000 & 0.003 & 99.998 \\
\hline 22 & 0.011 & 0.32 & 99.47 & 19 & 0.00 & 0.013 & 99.97 & 18 & 0.000 & 0.002 & 100.00 \\
21 & 0.009 & 0.25 & 99.72 & 21 & 0.00 & 0.012 & 99.98 & 19 & 0.000 & 0.000 & 100.00 \\
19 & 0.007 & 0.20 & 99.93 & 20 & 0.00 & 0.009 & 99.99 & & & & \\
\hline 1 & 0.001 & 0.04 & 99.96 & 22 & 0.000 & 0.004 & 99.997 & & & & \\
2 & 0.001 & 0.04 & 100.00 & 23 & 0.000 & 0.003 & 100.00 & & & & \\
\hline Sum & 3.440 & 100.0 & & Sum & 4.701 & 100.0 & & Sum & 1.856 & 100.0 & \\
\hline
\end{tabular}

Table 3. Variance decomposition for periods P3, P6 and P9.

period 5. Table 3 gives the contribution of each IMF to the total sum of variances for periods P3, P6 and P9 in absolute terms, percentage of total and cumulative percentage contribution. As can be seen, there is a large variation in the contribution from each IMF to the total variance. This can also be seen in Figure 4 right which shows the boxplots for the amplitude content of each IMF for periods 3, 6 and 9. Figure 5 shows the contribution (\%) of each IMF to the total variance for all periods. Only IMFs from 1 to 13 are considered. The main contribution to the total energy comes from IMFs 5 to 11, and this is in contrast to what has been reported previously by Veltcheva and Guedes Soares [7] and Veltcheva [8] where the first three IMFs are the most energetic ones. For example, in [7], IMF2 was found to be the most energetic followed by IMF3 
for two of the data sets, and for the other data set IMF1 is first followed by IMF2.

Schlurmann [9] analyses a transient wave recorded in the Sea of Japan and reports IMF2 as having the highest energy.

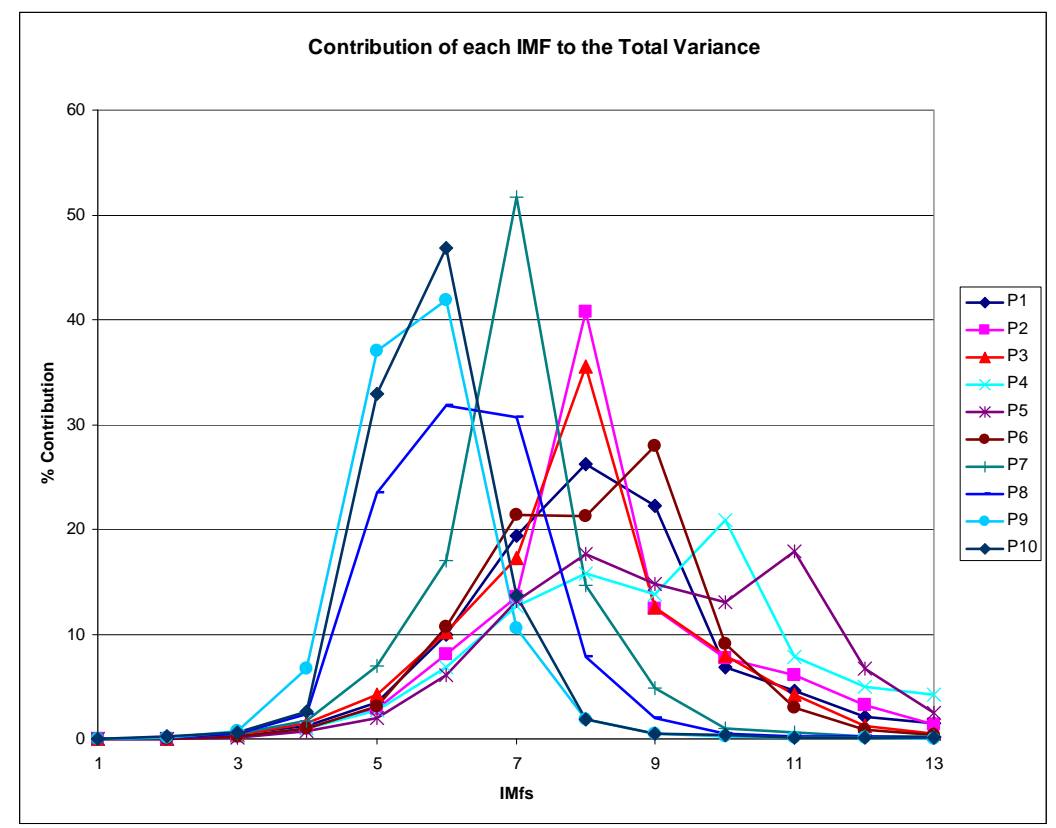

Figure 5: Contribution of each IMF to the Total Variance, all Periods.

\section{Spectral Analysis}

Using HHT-DPS one can obtain a graph for the Hilbert Energy Spectrum which has time and frequency as axes while the energy is shown in a colour scale. Figure 6 shows the Hilbert Spectrum for period P3. This graph provides a detailed record of the variation of amplitude in frequency and energy with time. For example, one can see that most of the energy is concentrated in a band around a frequency of $0.1 \mathrm{~Hz}$ and also that the amount of energy increases towards the end of the period, which is consistent with the significant wave height evolution (see Figure 1). Although there are high frequency components present in the spectrum, most of the energy is in the low frequencies, represented by IMFs 6, 7, 8 and 9 .

One of the alleged advantages of the HHT algorithm is that it is capable of revealing the local (time) behaviour of the analyzed signal. A section of the Hilbert Spectrum 


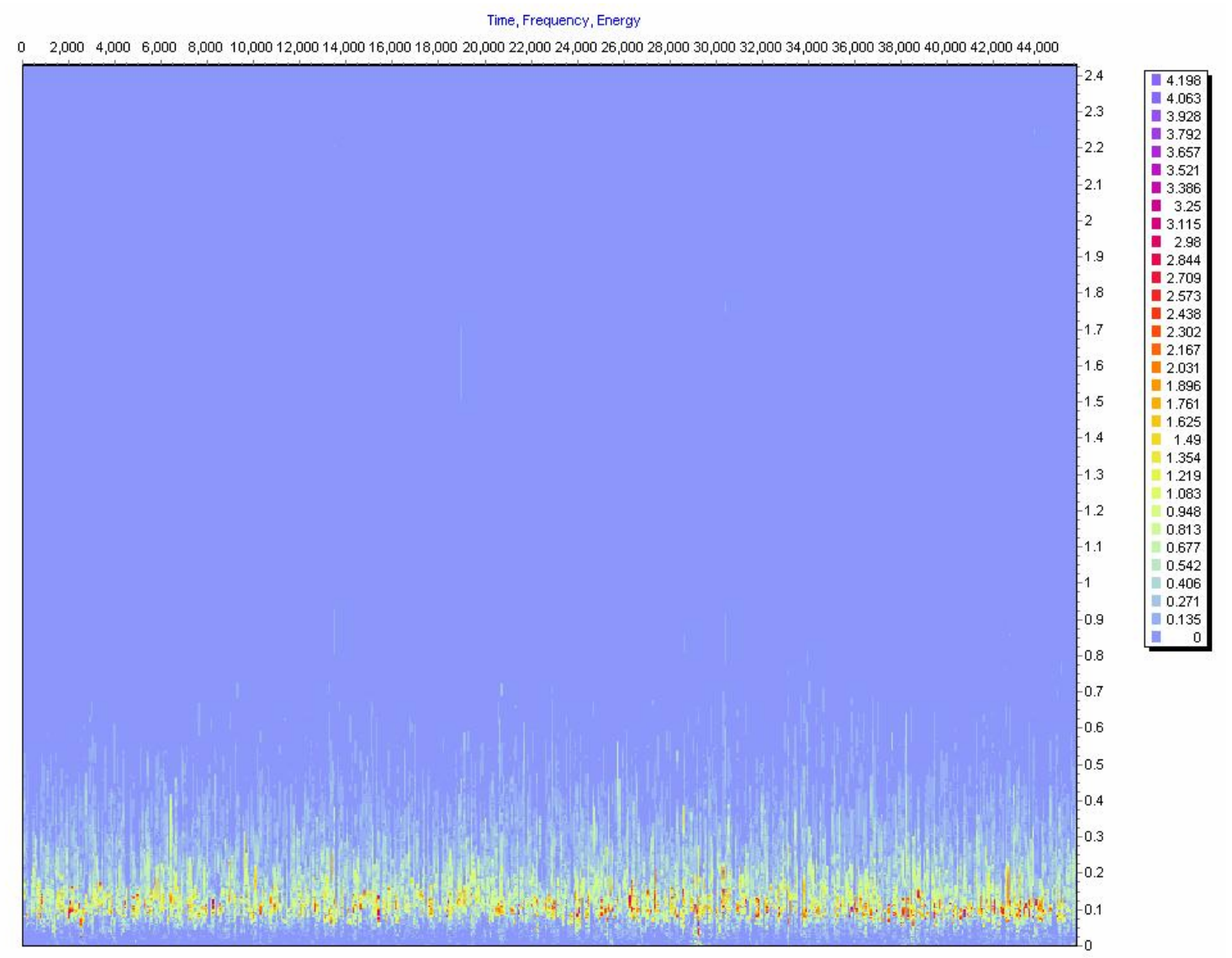

Figure 6. Hilbert Spectrum for Period 3.

corresponding to time points where large waves occurred during the storm was analyzed and compared with the shape of the wave-height record. In figure 7 we show the contour plot for the Hilbert spectra for a 200-seconds interval that contains two waves with amplitude over $10 \mathrm{~m}$. The first large wave occurs at about 40 seconds and is clearly identified by the Hilbert spectrum in which high amplitude is assigned to certain frequencies at the time the wave occurs. However, for the second wave, the amplitude is distributed in several frequencies as a consequence of the EMD. Figure 8 illustrates the low frequencies over a shorter time period for both waves. The degree to which the IMFs represented a decomposition of the original time variation was checked by adding the individual IMFs and comparing with the original time series. The reconstruction obtained is very precise, with an absolute error below $5 \times 10^{-13}$.

Looking closer at the decomposition for both large waves one can see that wave 1 (Fig. 9, left) is mainly the product of the superposition of a small number (5) of IMFs with 


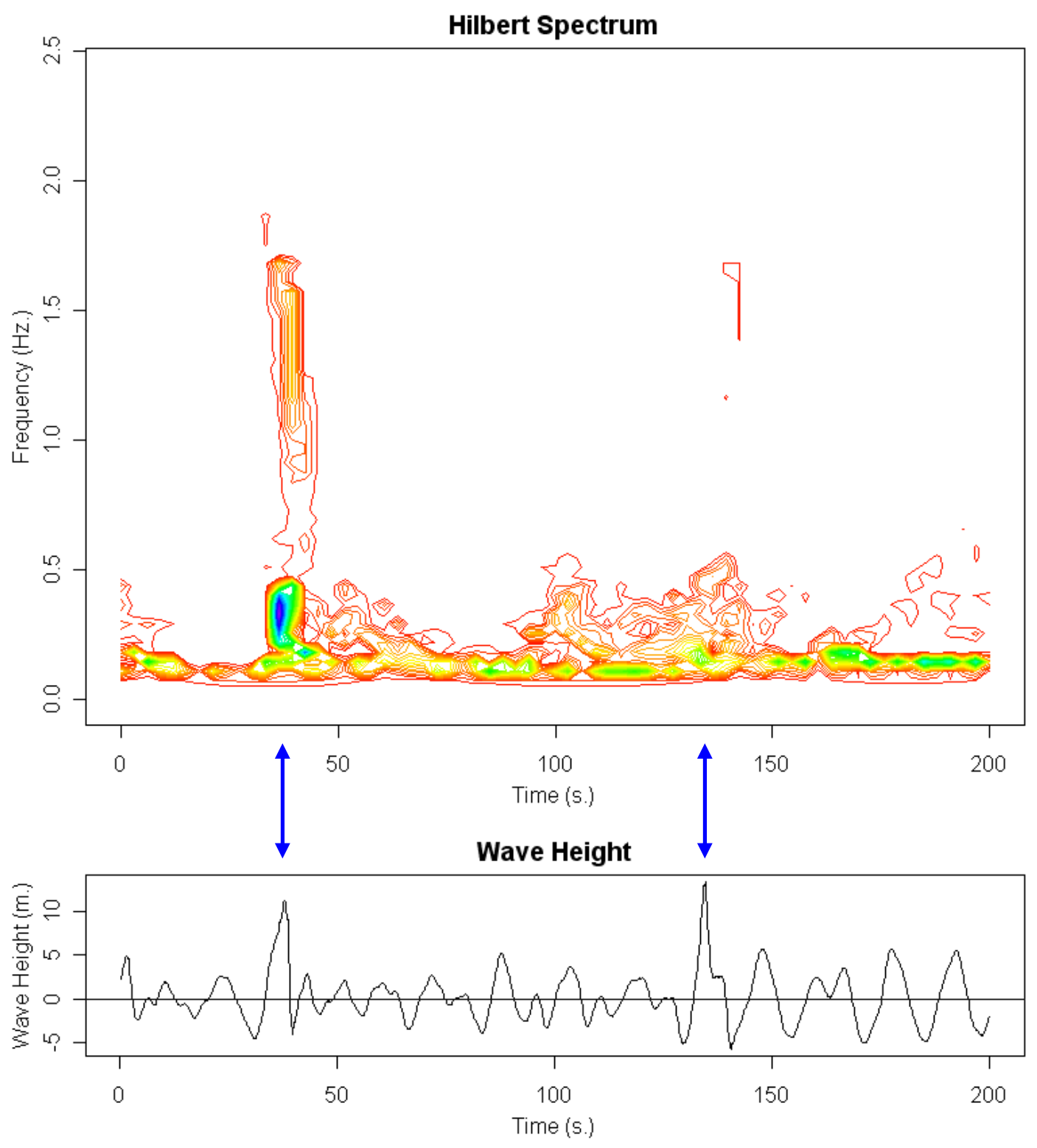

Figure 7. Hilbert spectrum for a 200 second interval with two big waves.

large amplitudes, while wave 2 (Fig. 9, right) is the result of the superposition of a larger number (9) of IMFs having moderate amplitudes that fall into phase at the moment the large wave is produced. For wave 2 none of the IMFs has amplitude above $3 \mathrm{~m}$.

\section{Comparison with Fourier Spectra}

The Fourier spectra from all 10 periods were considered, but we only present the results for Period 3. The spectra for all IMFs along with that from the original data was 

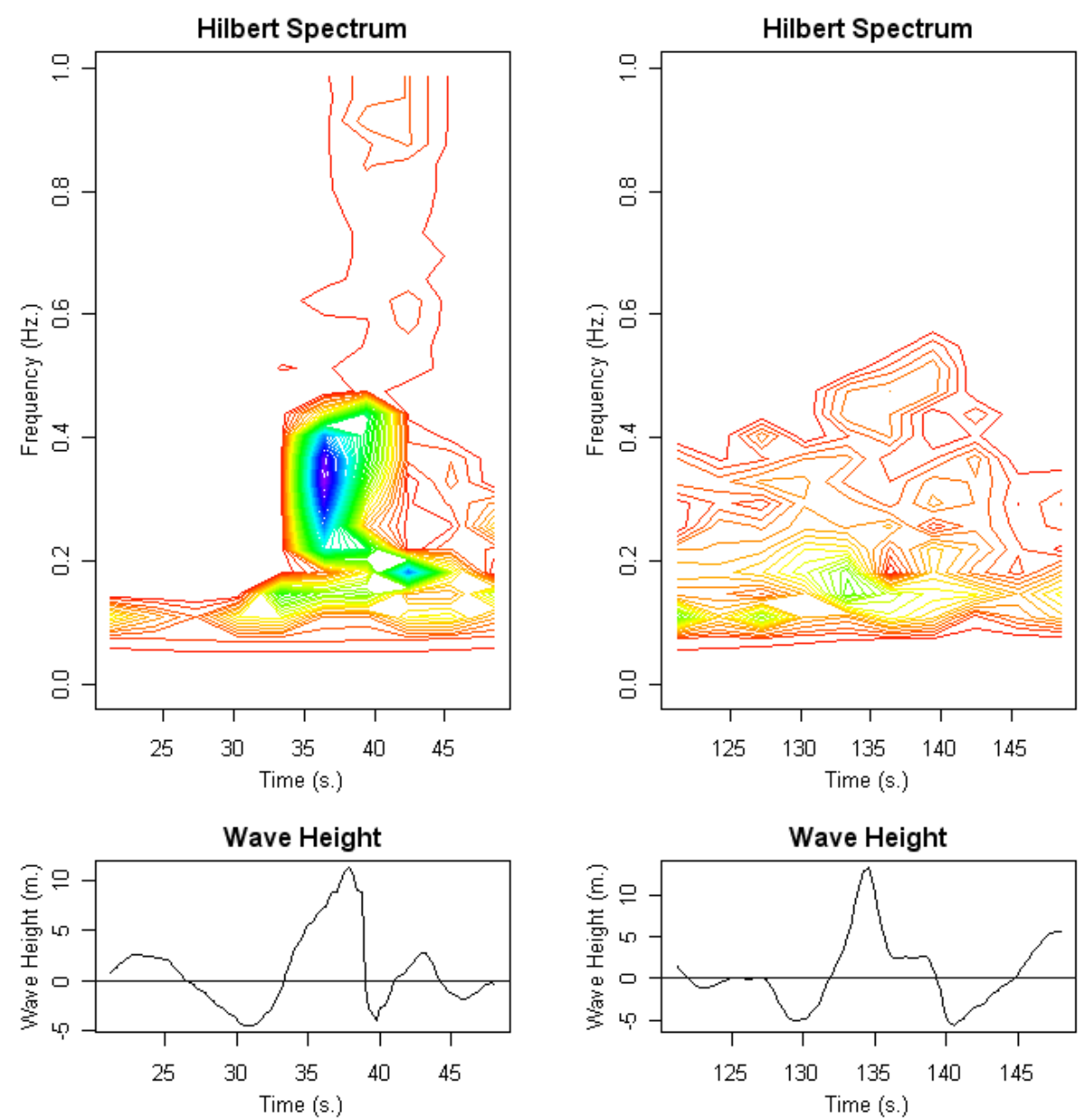

Figure 8. Hilbert spectra for two big waves.
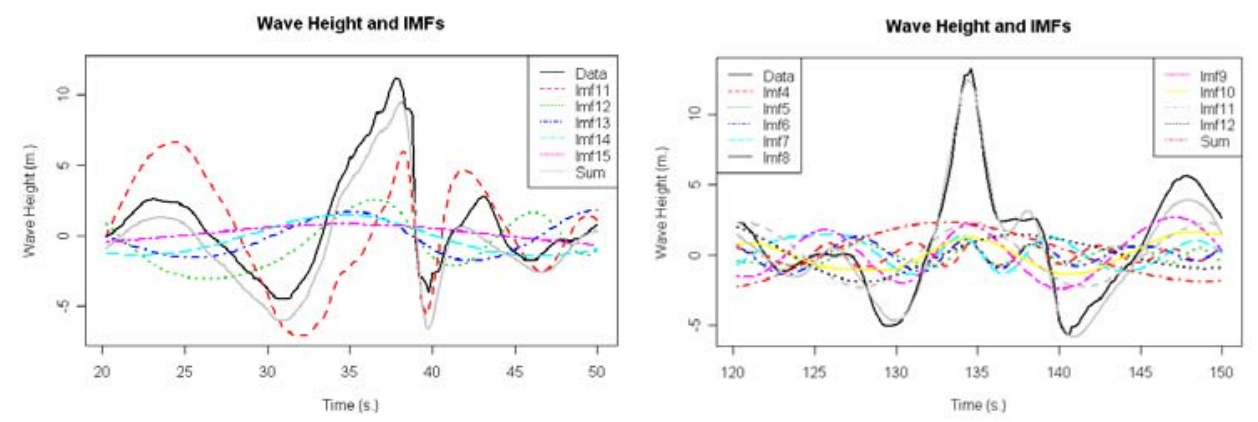

Figure 9. Wave height and 5 IMFs for waves 1 and 2.

number increases the corresponding frequency range and peak frequencies decrease, although for higher IMFs (17 and higher) the peak period remains almost constant. Also, the energy order coincides with that given in Table 3 with IMFs 6 to 10 having the greatest energy level and a peak frequency similar to that of the whole data set. Table 4 lists some of the important spectral characteristics obtained from the Fourier spectra; significant wave height (Hs), mean wave period (T01) and peak period (Tp) for 


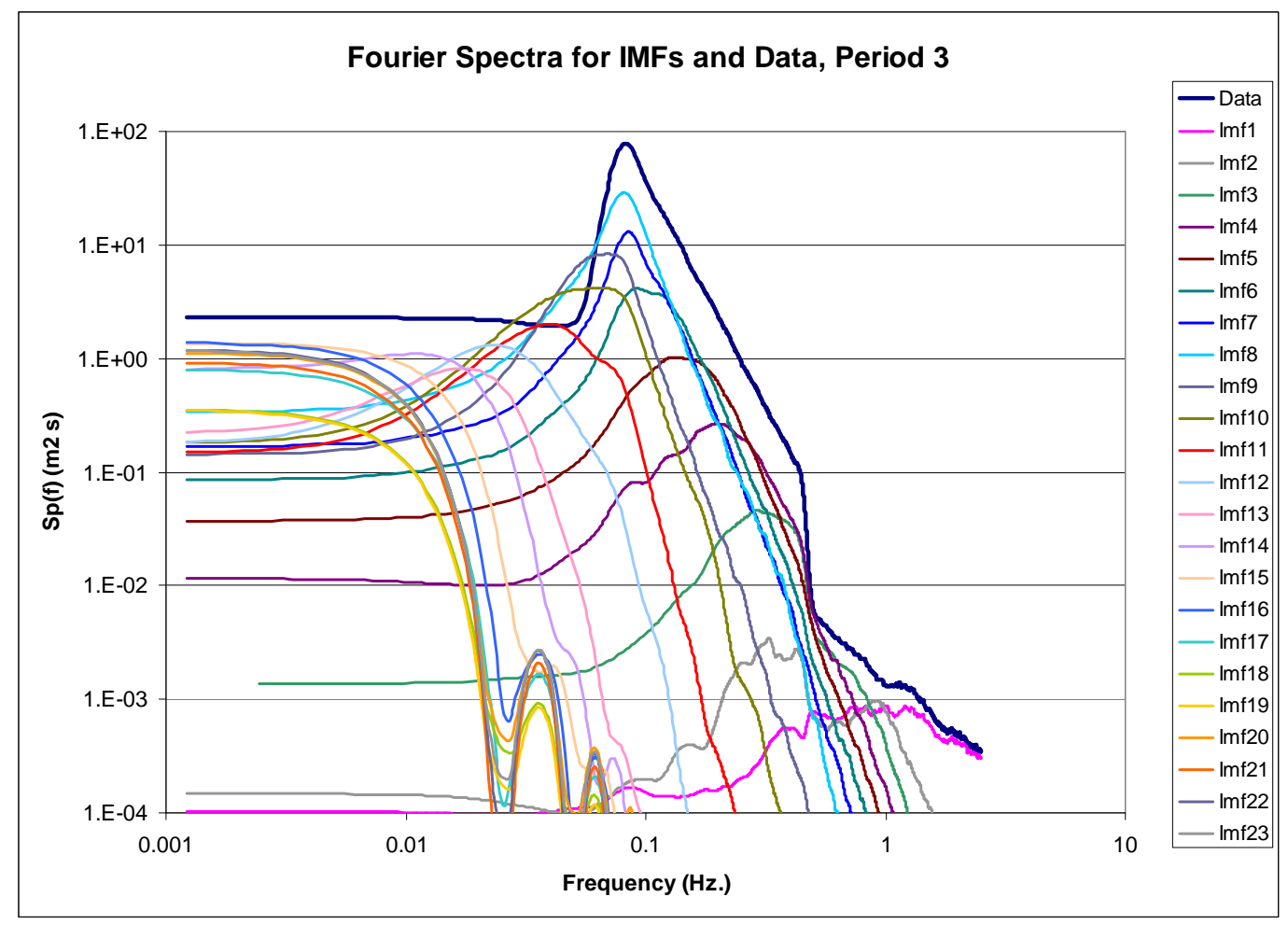

Figure 10. Fourier Spectra for IMFs and Data, Period 3.

\begin{tabular}{|c|r|r|r|r|r|r|r|r|}
\cline { 2 - 9 } \multicolumn{1}{c|}{} & \multicolumn{1}{c|}{ Data } & Imf 1 & Imf 2 & Imf 3 & Imf 4 & Imf 5 & Imf 6 & Imf 7 \\
\hline Hs (m) & 7.33 & 0.14 & 0.14 & 0.43 & 0.92 & 1.52 & 2.37 & 3.08 \\
\hline Tm01 (s) & 9.6 & 0.82 & 1.6 & 2.96 & 4.34 & 6.03 & 8.29 & 10.16 \\
\hline Tp (s) & 11.9 & 0.96 & 2.79 & 3.17 & 4.84 & 6.87 & 9.69 & 11.6 \\
\hline \hline & Imf 8 & Imf 9 & Imf 10 & Imf 11 & Imf 12 & Imf 13 & Imf 14 & Imf 15 \\
\hline Hs (m) & 4.42 & 2.62 & 2.08 & 1.53 & 0.83 & 0.54 & 0.71 & 0.56 \\
\hline Tm01 (s) & 11.52 & 14.14 & 15.9 & 25.04 & 35.1 & 53.1 & 82.1 & 128.2 \\
\hline Tp (s) & 12.2 & 14.8 & 16.5 & 49.95 & 43.0 & 57.8 & 138.2 & 253.0 \\
\hline \hline & Imf 16 & Imf 17 & Imf 18 & Imf 19 & Imf 20 & Imf 21 & Imf 22 & Imf 23 \\
\hline Hs (m) & 0.64 & 0.52 & 0.44 & 0.34 & 0.43 & 0.37 & 0.42 & 0.44 \\
\hline Tm01 (s) & 149.3 & 162.5 & 168.6 & 176.8 & 183.8 & 186.5 & 188.1 & 187.8 \\
\hline Tp (s) & 334.7 & 355.7 & 363.1 & 366.4 & 368.2 & 368.8 & 369.1 & 369.1 \\
\hline
\end{tabular}

Table 4. Spectral Characteristics for the IMFs and Data, Period 3.

the original data and for all IMFs. Except in very few cases the periods are seen to be increasing with the IMFs.

Figure 11 gives the marginal Hilbert spectra (obtained as a time integral of the Hilbert energy spectra) for the original data and the IMFs. These spectra have a different interpretation. In the Fourier case the process is assumed to be stationary and linear and the presence of energy associated to a given frequency means that there is a trigonometric component with this frequency and amplitude present during the whole 
time span of the data. In contrast, for the Hilbert marginal spectrum the process need not be stationary nor linear and the presence of energy associated to a given frequency means that in the time span of the data, there is a probability proportional to the amount of energy of having a component with this frequency and amplitude at any time.

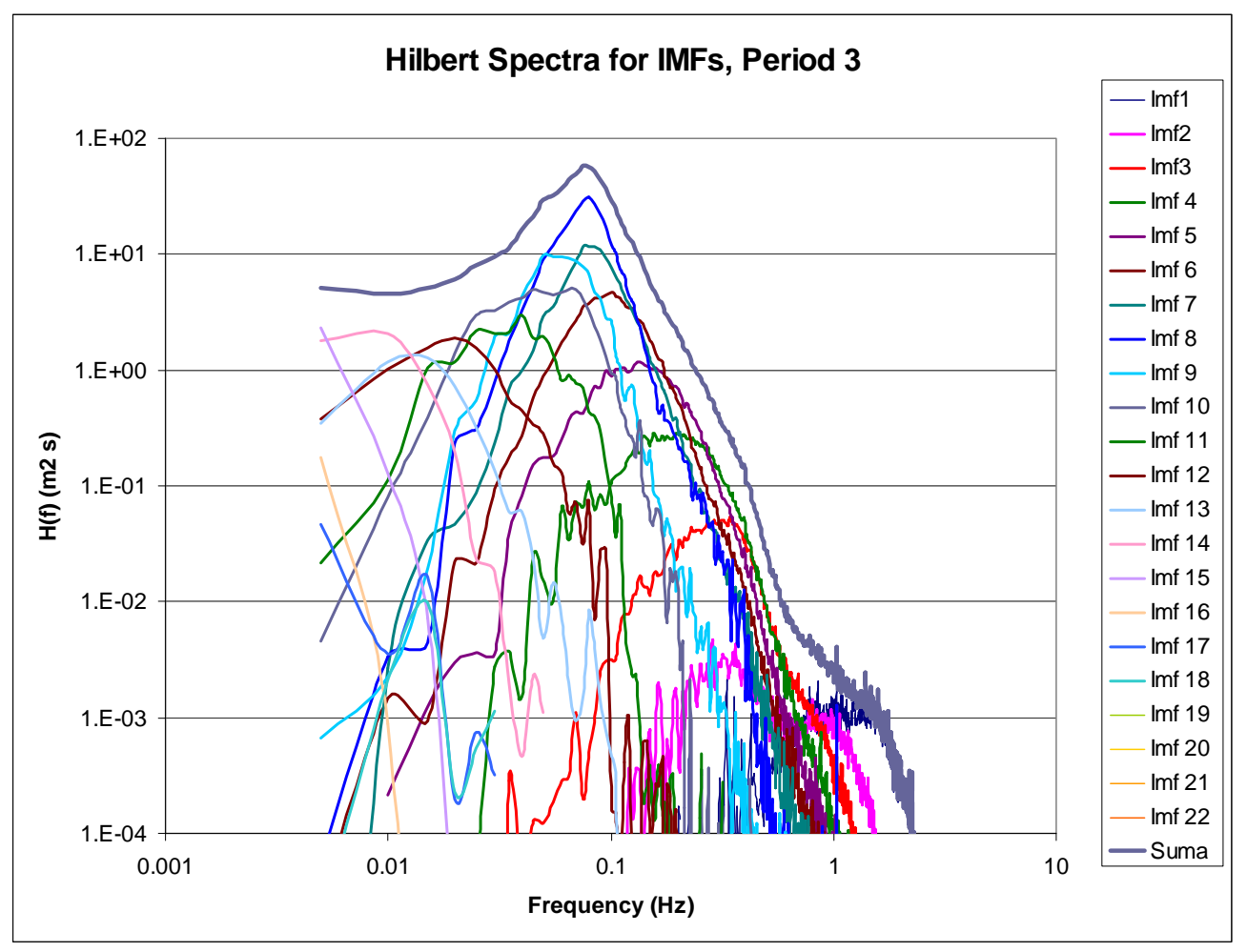

Figure 11. Hilbert Marginal Spectra for IMFs and Data, Period 3.

IMFs are supposed to represent different oscillatory modes present in the original data, each having different associated energies. This can be seen both from the Hilbert and the Fourier spectra. The first IMFs cover the high frequency range and have increasing energy as the IMF number grows. The central part of the spectrum is covered by IMFs 6 to 10 , which have roughly similar peak frequency. In this case the energy grows up to IMF 8 and then decreases. Higher IMFs decrease in frequency and energy, the last few being concentrated in the very low frequency range but also with low energy.

\section{Conclusions}

We have carried out a spectral analysis for a data set coming from a storm in the North 
Sea that took place in 1997, using the Hilbert-Huang Transform. The storm was decomposed into intervals of 12 hours or less and each data set was considered, although we only give results for ten 12-hour periods.

Each of these sets was decomposed into Intrinsic Mode Functions using HHT-DPS and their contribution to the total energy of the period was analyzed. The number of IMFs needed to decompose the data was higher than has been reported previously for different sea states. The amount of energy associated to each IMF was seen to vary for different time periods and reflects the specific characteristics of each period. However, it was seen that the energy distribution among different IMFs also differs from that reported previously. This may be due to the fact that sea states produced during a storm are more complex, and in particular the change in variance during the storm's evolution, requires more IMFs with a different energy distribution to be decomposed than that for normal, 'stationary' sea states. Moreover, the possibility of analyzing very long data sets sampled at high frequencies allows for the detection of frequencies in both extremes, high and low, that may not have been present in normal sea states, or which could not be detected in the data sets considered by other authors, usually sampled at around $1 \mathrm{~Hz}$ and for $20 \mathrm{~min}$. The influence of sampling rate and length of the data set on the EMD and the HHT in general requires further work but preliminary results obtained with the same data set suggests that they may have a high impact on both the number of IMFs required for signal decomposition and the energy distribution among the different IMFs.

Detailed analysis of two large waves occurring in the time series, showed that in one case, the Hilbert spectrum associates a large energy with the wave, if the wave is the result of the superposition of a single large, dominant IMF with a contribution from smaller ones. In the second case, when the large wave is the sum of several mediumsized EMFs, the Hilbert spectrum does not have a large amount of energy associated to 
the big wave. Although the IMFs do not always have a clear physical interpretation, these results could point to different mechanisms for generating large waves during a storm.

During a storm the state of the sea is clearly non-stationary. So it is expected that the Fourier analysis over a 12 hour period be unstable. On the other hand, the very sharp time resolution of the HHT procedure provides a more robust spectral analysis that is capable of providing information of the evolution of the data that is not possible with the conventional Fourier Spectrum.

\section{Acknowledgements}

The authors would like to thank Total E\&P UK for the wave data from the Alwyn North platform. The Hilbert-Huang Transform - Data Processing System is copyright United States Government as represented by the Administrator of the National Aeronautics and Space Administration and was used with permission. The software WAFO developed by the Wafo group at Lund University of Technology, Sweden was used for the calculation of all Fourier spectra and associated spectral characteristics. This software is available at http://www.maths.lth.se/matstat/wafo. This work was partially supported by CONACYT, Mexico, Proyecto Análisis Estadístico de Olas Marinas.

\section{References}

[1] Cohen, L. Time-frequency analysis. Englewood Cliffs: Prentice-Hall; 1995.

[2] Dätig, M., Schlurmann, T. 'Performance and limitations of the Hilbert-Huang transformation (HHT) with an application to irregular water waves,' Ocean Engineering 2004; 31: 1783-1834.

[3] Huang, N.E., Shen Z., Long S.R., Wu M.C., Shin H.S., Zheng Q., Yuen Y., Tung C.C., Liu H.H. “The empirical mode decomposition and Hilbert spectrum for nonlinear and non-stationary time series analysis,” Proc R Soc Lond, 1998; 454: 903-95. 
[4] Huang, N.E., Shen Z., Long S.R. “A new view of nonlinear water waves: the Hilbert spectrum,” Ann Rev Fluid Mech 1999; 31: 417-57.

[5] Huang, N.E., Wu M.L., Long S.R., Shen S.P., Per W.Q., Gloersen P., Fan K.L. “A confidence limit for the empirical mode decomposition and Hilbert spectral analysis,” Proc R Soc Lond 2003; 459: 2317-45.

[6] Huang, N.E. “Introduction to Hilbert-Huang Transform and some recent developments,” In: Huang N., Attoh-Okine N.O., editors. The Hilbert-Huang transform in Engineering. CRC Press, 2005; 1-23.

[7] Huang, N.E. "Introduction to Hilbert-Huang Transform and its related mathematical problems,” In: Huang N.E., Shen S.S.P., editors. Hilbert-Huang Transform and Its Applications. World Scientific, 2005; 1-26.

[8] Schlurmann, T. “The empirical mode decomposition and the Hilbert spectra to analyse embedded characteristic oscillations of extreme waves,” In: Rogue Waves, Editions Infremer, ISBN: 2-84433-063-0, 2000; 157-165.

[9] Schlurmann, T. "Spectral analysis of nonlinear water waves based on the HilbertHuang Transformation,” Journal of Offshore Mechanics and Arctic Engineering, American Society of Mechanical Engineers (ASME) 2002; 124 (1), 22-27.

[10] Veltcheva, A.D., Guedes Soares, C. "Identification of the components of wave spectra by the Hilbert Huang transform method,” Appl Ocean Res. 2004; 26: 1-12.

[11] Veltcheva, A.D. “An application of HHT method to the nearshore sea waves,” In: Huang N., Attoh-Okine N.O., editors. The Hilbert-Huang transform in Engineering. CRC Press, 2005; 97-119.

[12] Wolfram, J., Feld, G., Allen, J. “A new approach to estimating environmental loading using joint probabilities,” 7th Int. Conf. on Behaviour of Offshore Structures, Pergamon, Boston, 1994; Vol. 2: 701-713. 